\title{
Re-Enclavation of a Dislocated Retropupillary Fixated Iris Claw Lens: A Case Report
}

\section{Christoph Leisser Oliver Findl}

Vienna Institute for Research in Ocular Surgery, A Karl Landsteiner Institute, Hanusch Hospital, Vienna, Austria

\section{Keywords}

Intraocular lens dislocation $\cdot$ Re-enclavation $\cdot$ Retropupillary fixated iris claw lens

\begin{abstract}
A male patient, 63 years of age, presented with dislocation of a retropupillary fixated iris claw lens due to nasal haptic disenclavation. During re-enclavation the intraocular lens dislocated again. To avoid further dislocation, both superior fingers of the haptics were pushed through the iris tissue to achieve more stable support of the haptic ends. This method of fixation appeared to be well tolerated.

(C) 2020 The Author(s)

Published by S. Karger AG, Basel
\end{abstract}

\section{Introduction}

Retropupillary enclavation of iris claw lens, first described by Amar in 1980 [1], has become a common option of restoring vision in patients without capsular support. A modified technique of implantation was published by Mohr et al. [2] and is a reasonable alternative to the conventional enclavation of the iris claw lens from the anterior side. However, there is risk of loss of enclavation with both types of fixation resulting in subluxation of the intraocular lens $[3,4]$. We present a technique of re-enclavation of a retropupillary positioned iris claw lens that had a tendency for repeated disenclavation. 


\section{Case Report}

A male patient, 63 years of age, presented to our outpatient department with decreased visual acuity. During examination, loss of enclavation at the nasal haptic was discovered, resulting in dislocation of a retropupillary positioned iris claw lens that had been implanted 17 months before (Artisan Aphakia Model 205; Ophtec BV, Groningen, The Netherlands). The lens was successfully lifted with a spatula via a 23G sclerotomy and re-enclavation was performed using a spatula to push iris tissue into the lobster claw of the lens. Immediately, loss of enclavation happened again. It was decided to push the superior fingers of both iris claw haptics through the iris tissue. This resulted in stable positioning without detectable irritation of the tissue.

Postoperative best distance corrected visual acuity was 0.6 (Snellen charts) at the followup 6 days after surgery. The re-enclavated iris claw lens was at the designated position at the backside of the iris (Fig. 1), with no sign of tilt or significant anterior segment inflammation.

\section{Discussion}

Loss of enclavation of iris claw lenses, a rare postoperative finding in this kind of surgery $[3,4]$, seems to be caused by iris tissue alterations, such as atrophy, as in cases of spontaneous disenclavation of iris claw lenses the closure of haptics was examined to be sufficient compared to cases with traumatic disenclavation [5].

Re-enclavation with pushing the superior fingers of both iris claw haptics through the iris tissue appears to be a safe option for patients with dislocated retropupillary iris claw lenses and reduces the need for lens exchange.

\section{Statement of Ethics}

Written informed consent was obtained from the patient for publication of his case.

\section{Disclosure Statement}

O. Findl is a scientific advisor for Carl Zeiss Meditec AG, but has no personal interest in the products mentioned. Both authors declare that there are no conflicts of interest.

\section{Funding Sources}

There was no funding.

\section{Author Contributions}

C. Leisser: conception of the study, data acquisition, manuscript preparation. O. Findl: conception of the study, data acquisition, manuscript preparation, critical review of the manuscript. 


\section{Case Reports in Ophthalmology}

\section{References}

1 Amar L. Posterior chamber iris claw lens. Am Intra Ocular Implant Soc. 1980;6:27.

2 Mohr A, Hengerer F, Eckardt C. Retropupillary fixation of the iris claw lens in aphakia. 1 year outcome of a new implantation techniques. Ophthalmologe. 2002 Jul;99(7):580-3. German.

3 Gonnermann J, Klamann MK, Maier AK, Rjasanow J, Joussen AM, Bertelmann E, et al. Visual outcome and complications after posterior iris-claw aphakic intraocular lens implantation. J Cataract Refract Surg. 2012 Dec;38(12):2139-43.

4 Forlini M, Soliman W, Bratu A, Rossini P, Cavallini GM, Forlini C. Long-term follow-up of retropupillary irisclaw intraocular lens implantation: a retrospective analysis. BMC Ophthalmol. 2015 Oct;15(1):143.

5 Brockmann T, Gonnermann J, Brockmann C, Torun N, Joussen AM, Bertelmann E. Morphologic alterations on posterior iris-claw intraocular lenses after traumatic disenclavation. Br J Ophthalmol. 2014 Sep;98(9):13037.

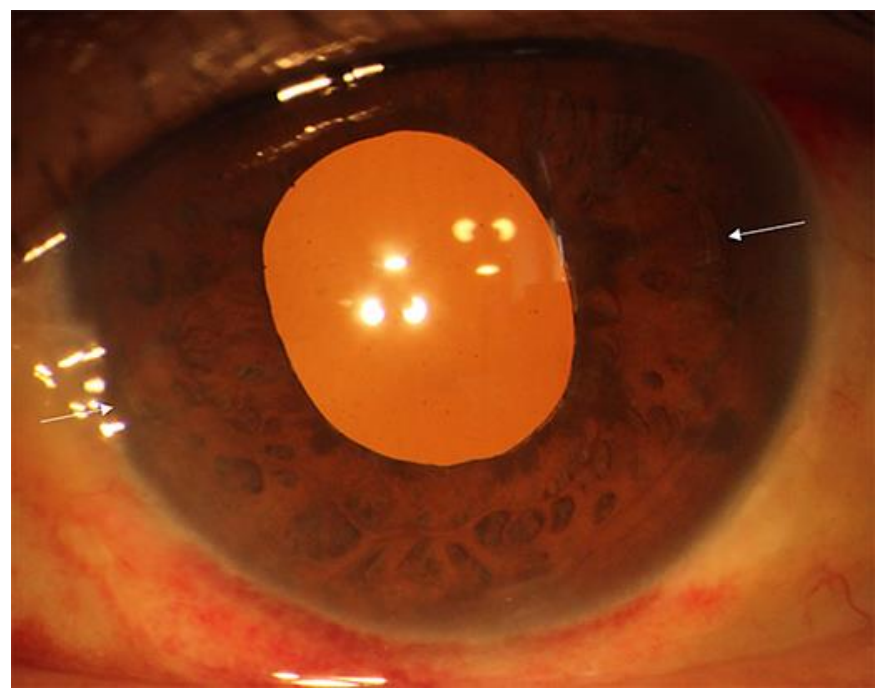

Fig. 1. The superior fingers of the iris claw haptics were pushed through the iris tissue and now offered enough support for a stable re-enclavation (arrow). 